\title{
Clinical manifestations of headache in children younger than 7 years
}

Bu Seon Kang ${ }^{1}$, Jinsun Lee, MD², Jin Hyuk Choi, MD², Hyeok Hee Kwon ${ }^{3}$, Joon Won Kang MD, PhD²,3,

${ }^{1}$ Department of Medicine, Chungnam National University School of Medicine, Daejeon, ${ }^{2}$ Department of Pediatrics, Chungnam National University Hospital, Chungnam National University School of Medicine, Daejeon, ${ }^{3}$ Department of Medical Science, College of Medicine, Chungnam National University, Daejeon, ${ }^{4}$ Brain Research Institute, Chungnam National University School of Medicine, Daejeon, Korea

Purpose: Headache is a common symptom during childhood. It is usually persistent and requires special care. This study aimed to identify the characteristics of headache in children $<7$ years of age.

Methods: We reviewed 3 years of clinical files on children $<7$ years of age with a chief complaint of headache.

Results: This study included 146 children (66 males, 80 females; mean age, $5.5 \pm 1.0$ years). Mean symptom duration was $5.8 \pm 7.9$ months. Attack durations were longer than 2 hours in 31 patients, shorter than 2 hours in 70 patients, and unchecked in 45 patients. Attack frequency was $15.1 \pm 10.6$ times per month. Pain locations and characteristics were also variable. Mean pain severity score was $5.1 \pm 2.2$ on the visual analog scale. Of 38 patients who underwent electroencephalography, 9 showed positive findings. Of 41 who underwent brain magnetic resonance imaging, 20 showed positive findings. The diagnoses were migraine (including probable migraine) in 34, tension-type headache in 5, and congenital malformations in 3. Medications were used in 29 patients: acetaminophen in 17, ibuprofen in 8, naproxen sodium in 1 , and topiramate or amitriptyline in 3.

Conclusion: In children aged $<7$ years, headache has a relatively benign course, but detailed history taking is needed for more accurate diagnosis.

Key words: Headache, Child, Migraine

\section{Introduction}

Headache is considered one of the most common symptoms during childhood that is usually long-lasting and frequent enough to need special care. ${ }^{1)}$ The lifetime prevalence of headache in childhood and adolescence is estimated to be approximately $40 \%$ in the general pediatric population, as compared to 4\%-20\% in preschool children and 38\%-50\% in school-aged children. ${ }^{2}$ There are considerable differences between the headaches of children and adults in terms of pain duration, laterality, and other factors. ${ }^{3)}$ In preschool children, the attack duration was shorter with lower symptom association, and it showed greater male gender prevalence and earlier onset of the attacks. ${ }^{3-5)}$

Distinctions exist even among children by age. Headache in preschoolers are considered relatively less common. Previous studies reported that headache prevalence in children 3-4 years of age was 3\%-6\% and increased from 5.9\% to 6.3\% around 7 years of age. ${ }^{4,5)}$ Additional studies and diagnostic criteria specific to preschoolers are necessary because of difficulties of children under 6 years of age have describing the pain precisely during clinical examination. ${ }^{4}$ Therefore, more workups in various perspectives are needed to determine the accurate aspects of headache in preschoolers. Most are primary headaches without a specific disease,
Corresponding author: Joon Won Kang, MD, PhD Department of Pediatrics, Chungnam National University Hospital, Chungnam National University School of Medicine, 282 Munwha-ro, Jung-gu, Daejeon 35015, Korea

Tel: +82-42-280-7244

Fax: +82-42-255-3158

E-mail: childlove@cnu.ac.kr

https://orcid.org/0000-0001-5756-3814

Received: 12 February, 2018

Revised: 30 June, 2018

Accepted: 16 July, 2018

Copyright (c) 2018 by The Korean Pediatric Society

This is an open-access article distributed under the terms of the Creative Commons Attribution NonCommercial License (http://creativecommons.org/ licenses/by-nc/4.0/) which permits unrestricted noncommercial use, distribution, and reproduction in any medium, provided the original work is properly cited. 
whereas secondary headaches arise due to organic diseases mostly manifesting other symptoms. ${ }^{6}$ In these contexts, the objective of the present study was to examine various aspects and progression of headaches in children younger than 7 years.

\section{Materials and methods}

\section{Study population and data source}

Between 2013 and 2015, a total of 748 patients (390 males, 358 females) visited the Department of Pediatrics at Chungnam $\mathrm{Na}$ tional University Hospital complaining of headache. Among them, 146 patients (19.5\%; 66 males, 80 females) were younger than 7 years old. In addition to their demographic characteristics, some features such as age at the visit and follow-up period duration were retrospectively checked from the medical records.

The clinical data were also retrospectively collected from the medical records in this study. These data involve characteristics of headache including disease duration, attack frequency, attack duration, pain location, related symptoms, pain severity, and family history of headache. It was obtained with the help of the guardian because it was difficult to find out the characteristics on its own. In the case of the item with time concept, it was obtained by the supervisor's observation. In case of subjective contents such as headache or accompanying symptoms were obtained by the patient. The present study investigated the applicability of the International Classification of Headache Disorders (ICHD) 3-beta criteria for the patients' headaches. Data including height, body weight, blood pressure, laboratory tests, electroencephalography (EEG), brain magnetic resonance imaging (MRI) were also collected. The indications for investigations were: (1) abnormal neurologic examination, (2) abnormal or focal neurologic signs or symptoms, (3) seizures or very brief auras (<5 minutes), (4) unusual headaches in children (atypical auras, trigeminal autonomic cephalagia), (5) headache in children younger than 6 years old or any child who cannot adequately describe his or her headache, (6) brief cough headache, (7) headache worst on first awakening or that awakens the child from sleep, and (8) migrainous headache in the child with no family history or migraine. The positive findings, which indicate any abnormal results in EEG or MRI, were arranged more closely in specific outcomes despite some overlapping findings. All the interpretation of EEG was done by certificated pediatric neurologist. All the interpretation of MRI was done by certificated pediatric radiologist. Diagnosis, medication, and prognosis were also collected and organized. This study was approved by the Institutional Review Board of Chungnam National University Hospital (2017-11-048). Informed consent was exempted for all subjects due to the retrospective study nature.

\section{Statistical analysis}

The statistical analyses were done by SPSS ver. 17.0 (SPSS Inc., Chicago, IL, USA). Chi-square tests, and t test were applied to the data to compare variables; $P<0.05$ was regarded statistically significant.

\section{Results}

\section{Features of the study patients}

A total of 146 patients (66 males, 80 females) younger than 7 years old were included in this study. The average patient age at the visit was 5.5 \pm 1.0 years (range, 2.8-6.9 years). Fig. 1 shows that only $1.4 \%$ of the patients were younger than 3 years, $11.6 \%$ were 3-4 years, $15.1 \%$ were $4-5$ years, 33.6\% were 5-6 years, and 38.3\% were 6-7 years old. The older the age group, the higher percentage of male patients within it; in fact, the percentage of male patients increased steadily from $25 \%$ in the $3-4$ years of age group to $48.2 \%$ in the 6-7 years of age group (Fig. 1). The mean follow-up period was 22.0 \pm 9.8 months (range, 5.0-37.4 months) from the investigation day. The patients' height, weight, and blood pressure were in the normal range.

\section{Headache characteristics}

The mean disease duration was 5.8 \pm 7.9 months (range, 1 day to 3 years). The results showed that 36 patients (24.7\%) reported having headaches for 1-3 months, 33 (22.6\%) described having headaches for $>12$ months, and $2(1.4 \%)$ who did not specify the disease duration. The average attack frequency was 15.1 \pm 10.6 times per month. A total of 55 patients (37.7\%) reported having headaches more than once per week. Having headaches daily was recorded by 41 patients (28.1\%), while 32 patients (21.9\%) did not specify attack frequency. Additionally, regarding attack duration, the percentages

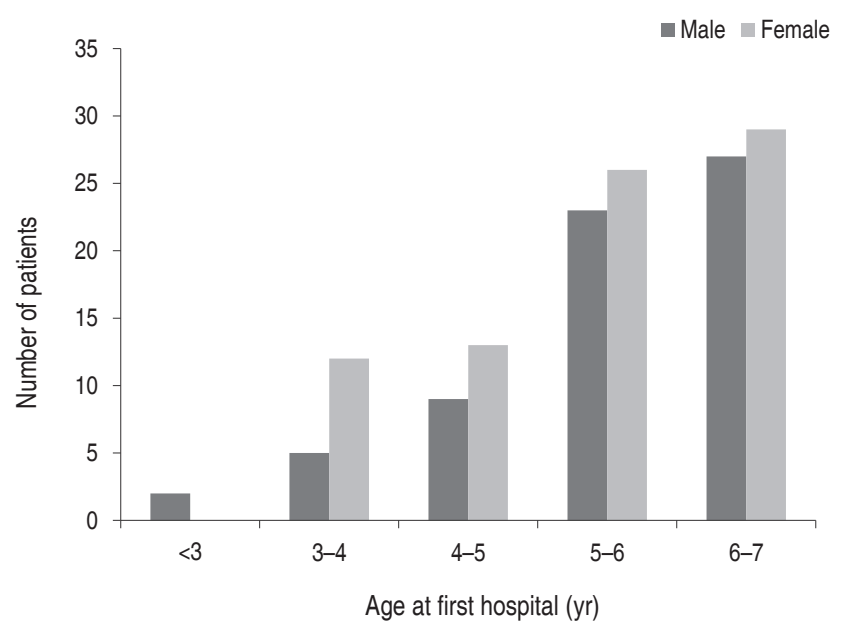

Fig. 1. Age at first hospital visit according to sex, based on record review at Chungnam National University Hospital, 2013-2015. 
each time group occupied were distributed quite evenly, ranging from $11.6 \%$ to $18.5 \%$, except for headaches $>72$ hours (3.4\%) (Table 1). Most patients (47.9\%) reported an attack duration of $<2$ hours and 21.2\% complained of headache from 2 hours to $>3$ days. A total of $30.9 \%$ of the patients could not clearly explain the duration. Expression of headache was stabbing in 26, squeezing in 21, throbbing in 16, dizziness in 3, others in 8 and unexplained in 72 patients.

Pain location was reported as unilateral fronto-temporal $(n=39$,

Table 1. Properties of headache reported by patients

\begin{tabular}{|c|c|}
\hline Variable & Value \\
\hline Disease duration (mo) & $5.8 \pm 7.9(0.03-36)$ \\
\hline$<2$ Weeks & 29 (19.9) \\
\hline 2-4 Weeks & $11(7.5)$ \\
\hline 1-3 Months & $36(24.7)$ \\
\hline 3-6 Months & $17(11.6)$ \\
\hline 6-12 Months & 18 (12.3) \\
\hline$\geq 12$ Months & $33(22.6)$ \\
\hline Unexplained & $2(1.4)$ \\
\hline Attack frequency (/mo) & $15.1 \pm 10.6$ \\
\hline Daily & $41(28.1)$ \\
\hline$\geq 1 /$ wk & $55(37.7)$ \\
\hline$<1 /$ wk & $18(12.3)$ \\
\hline Unexplained & $32(21.9)$ \\
\hline \multicolumn{2}{|l|}{ Attack duration } \\
\hline Few seconds & $26(17.8)$ \\
\hline$<30$ Minutes & $17(11.6)$ \\
\hline $0.5-2$ Hours & 27 (18.5) \\
\hline 2-72 Hours & $26(17.8)$ \\
\hline$\geq 72$ Hours & $5(3.4)$ \\
\hline Unexplained & $45(30.9)$ \\
\hline \multicolumn{2}{|l|}{ Pain location } \\
\hline Unilateral fronto-temporal & $39(26.7)$ \\
\hline Bilateral fronto-temporal & $23(15.8)$ \\
\hline Parietal & $13(8.9)$ \\
\hline Occipital & $11(7.5)$ \\
\hline Unexplained & $60(41.1)$ \\
\hline \multicolumn{2}{|l|}{ Related symptoms } \\
\hline Nausea/vomiting & $54(37)$ \\
\hline Photophobia/phonophobia & $31(21.2)$ \\
\hline Dizziness & $22(15.1)$ \\
\hline None & $61(41.8)$ \\
\hline Pain severity (visual analog scale) & $5.1 \pm 2.2$ \\
\hline Unexplained & $55(37.7)$ \\
\hline \multicolumn{2}{|l|}{ Family history of headache } \\
\hline Positive & $41(28.1)$ \\
\hline Negative & 105 (71.9) \\
\hline
\end{tabular}

Values are presented as mean \pm standard deviation (range) or number (\%).
26.7\%), bilateral fronto-temporal ( $n=23,15.8 \%)$, parietal $(n=13,8.9$ $\%$ ), or occipital ( $n=11,7.5 \%)$. Accumulating the reports of unilateral and bilateral pain location, a total of 62 patients (42.5\%) had lateralized headaches. Some patients reported having the pain in the parietal (8.9\%) or occipital (7.5\%) sites, while 60 patients (41.1\%) did not specify the pain location.

Regarding related symptoms, 54 patients (37.0\%) reported nausea or vomiting, followed by 31 (21.2\%) with photophobia or phonophobia and 22 (15.1\%) with dizziness. A total of 61 patients (41.8\%) had no associated symptoms. Mean pain severity on the visual analog scale (VAS) was 5.1 \pm 2.2 . However, 55 patients (37.7\%) could not explain their pain severity. In addition, only 41 patients (28.1\%) had any family history of headache, while 105 patients (71.9\%) had no family history.

\section{Laboratory findings}

The patients underwent blood tests such as complete blood count and thyroid function (Supplementary Table). One patient was specifically found to have an increased thyroid stimulating hormone (TSH) level of $12.62 \mu \mathrm{IU} / \mathrm{mL}$, so follow-up was decided for the patient. Her follow-up thyroid test results were as follows: TSH, $9.9 \mu \mathrm{IU} / \mathrm{mL}$; free thyroxine, $1.04 \mathrm{ng} / \mathrm{dL}$; and triiodothyronine, 1.03 $\mathrm{ng} / \mathrm{mL}$. She was diagnosed with subclinical hypothyroidism by a pediatric endocrinologist. Otherwise, there were no unusual clinical findings.

\section{Brain MRI and EEG findings}

Brain MRI was performed for 41 patients (28.1\%), among whom $48.8 \%$ had positive findings. With more details, the results showed that 3 patients had congenital malformation, including moyamoya disease, Chiari 1 malformation, and cavernous malformation. Two patients (10.0\%) with cavum septum pellucidum or cavum vergae and 1 patient with a pineal cyst were considered normal variation rather than congenital malformation. Additionally, 15 patients (75.0\%) had sinusitis or otomastoiditis. Some patients had more than 1 positive finding, so there are some overlapping numbers of the findings in Table 2. EEG was performed for 38 patients (26.1\%); among them, 23.7\% had positive findings. The results are described in Table 2. There were 5 patients with focal slowing waves, 4 with a slow and disorganized background, and 2 with epileptiform discharge.

\section{Classification of headache and outcomes}

Among the patients, 39 (26.7\%) were diagnosed with primary headache, 18 (12.3\%) received the diagnosis of secondary headache on ICHD 3-beta version, and 89 (61.0\%) were unclassified (Fig. 2). For more details, 16 (11.0\%) were diagnosed with migraine, 18 $(12.3 \%)$ received the diagnosis of probable migraine, and 5 patient (3.4\%) had tension-type headache among the patients with primary headache. In secondary headache, 15 patients (10.3\%) had 
Table 2. Patients' brain magnetic resonance imaging and electroencephalography results

\begin{tabular}{lc}
\hline Variable & No. (\%) \\
\hline Brain magnetic resonance imaging & \\
Not done & $105(71.9)$ \\
Done & $41(28.1)$ \\
Normal & $21(51.2)$ \\
Positive findings (overlapping) & $20(48.8)$ \\
Sinusitis/otomastoiditis & $15(36.6)$ \\
Cavum septum pellucidum/cavum vergae & $2(4.9)$ \\
Moyamoya disease & $1(2.4)$ \\
Chiari 1 malformation & $1(2.4)$ \\
Cavernous malformation & $1(2.4)$ \\
Pineal cyst & $1(2.4)$ \\
Unspecified cystic lesion & $1(2.4)$ \\
Unidentified bright object & $1(2.4)$ \\
Electroencephalography & \\
Not done & $108(73.9)$ \\
Done & $38(26.1)$ \\
Normal & $29(76.3)$ \\
Positive findings (overlapping) & $9(23.7)$ \\
Focal slowing & $5(13.2)$ \\
Slow and disorganized background & $4(10.5)$ \\
Epileptiform discharges & $2(5.3)$ \\
\hline
\end{tabular}

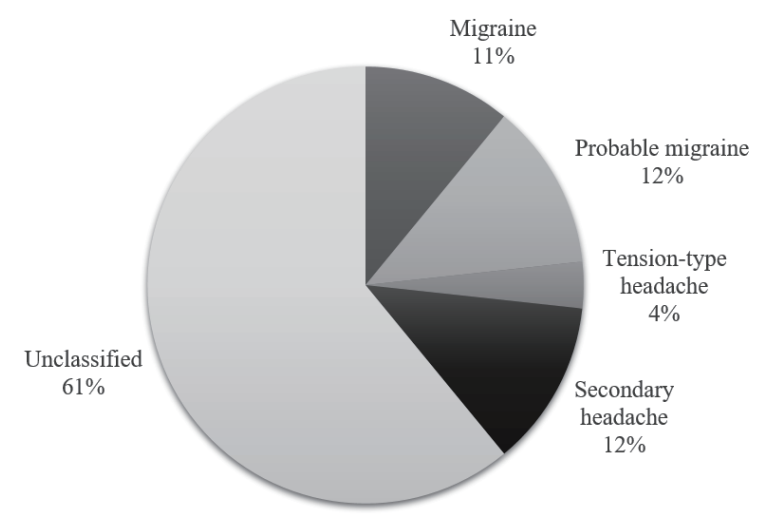

Migraine
- Secondary headache $\quad$ Unclassified

Fig. 2. Distribution of headache diagnosis, according to the International Classification of Headache Disorders 3rd edition - beta version, 2013.

a sinusitis, and 3 patients (2.1\%) had a congenital malformation. Other MRI positive findings were considered normal variations or nonspecific findings. A total of 29 patients (19.9\%) were treated with medication (Table 3); of them, 17 (11.6\%) used acetaminophen, 8 (5.5\%) used ibuprofen, and 1 (0.7\%) used naproxen sodium to control the acute pain. In addition, 2 (1.4\%) took topiramate and 1 (0.7\%) used amitriptyline. In terms of prognosis, 56.1\% of patients
Table 3. Diagnosis, management and prognosis of headache

\begin{tabular}{lc}
\hline Variable & No. (\%) \\
\hline Diagnosis & $39(26.7)$ \\
Primary headache & $16(11)$ \\
Migraine & $18(12.3)$ \\
Probable migraine & $5(3.4)$ \\
Tension-type headache & $18(12.3)$ \\
Secondary headache & $89(61)$ \\
Unclassified & \\
Management & $117(80.1)$ \\
None & $26(17.8)$ \\
Acute treatment & $17(11.6)$ \\
Acetaminophen & $8(5.5)$ \\
Ibuprofen & $1(0.7)$ \\
Naproxen & $3(2.1)$ \\
Prophylaxis & $2(1.4)$ \\
Topiramate & $1(0.7)$ \\
Amitriptyline & \\
Prognosis & $82(56.1)$ \\
Improved & $60(41.1)$ \\
Loss of follow-up & $4(2.8)$ \\
Consulted to neurosurgery &
\end{tabular}

showed improvement. Sixty patients were lost to follow-up after first hospital visit, 4 underwent consulted to the department of neurosurgery for congenital abnormalities, and unidentified bright lesion. There was no statistical significance between the headache characteristics and the test results according to the age group of the patients.

\section{Discussion}

Headache is a common finding in preschoolers. In this study, we analyzed the clinical features of these patients and found that the headaches were relatively benign. In the case of headache in preschool children, it is often difficult to listen to accurate history. However, since about 40\% of headache can be diagnosed according to ICHD 3-beta, clinician's efforts should be made for this. There are few studies about headache in preschoolers compared to schoolaged children. ${ }^{4,7,8)}$ Also, those studies mainly analyzed only primary headache or migraine, one of the most common diagnoses. This study did not limit participants to those with a specific diagnosis; rather, it included all patients with primary or secondary headache. We also focused on more specific age groups, so detailed aspects of headache in preschools could be investigated according to various factors.

Checking the results of this research in detail, the number of patients tended to gradually increase with age. Hernandez-Latorre and 
Roig ${ }^{9)}$ showed that more patients in the older groups experienced their first headache, while the number of patients with headache visiting the hospital increased with age until 7 years of age. There were only a few patients in the relatively younger groups, especially the group $<3$ years of age, even with no female patients. Parents may miss the expressions of headache that their young kid tries to show or misunderstand the specific symptoms shown by kids as just routine complains due to the linguistic limits of children that age.

With regard to the proportion of male to female patients with headache, Mavromichalis et al..$^{10)}$ showed that the sex ratio of patients with headache who are younger than 7-9 years was half and half, similar to Laurell et al. ${ }^{111}$ In the current study, the prevalence of headache in male patients consistently increased with age except in the age group $<3$, which included only 2 male and no female patients. In other words, the rate of male patients with headache was low in the younger groups, whereas that of female patients decreased gradually as age increased. Interestingly, there were a few suggestions in Korea such as the study of Hong et al. ${ }^{3)}$ with low ratio of female headache patients of younger ages. That article has some similarities with the current research: the patient data were collected in single Korean hospital for relatively short period and shared the Korean social stereotype that males should be braver than females. Those variables might have caused the differences in the male and female ratio in Korean reports compared to articles from other countries.

Mean disease duration was 5.8 \pm 7.9 months in this study. In addition to duration, other factors such as pain severity or frequency would affect the degree of suffering in daily life, which may lead headache patients to visit hospitals and obtain clinical treatment. Considering attack frequency first, in the current study, $65.8 \%$ of patients reported a headache more than 1 time per week, whereas 12.3\% experienced pain less than once per a week. Battistella et al. . $^{4}$ showed that $61 \%$ of preschoolers had more than 1 attack per week: 1-3 attacks per week in 47\%, and more than 4 attacks per week in $14 \%$. This result is similar to that of the present study in terms of the percentage with a frequency greater than half of all of the subjects.

The attack duration is generally shorter in younger children. ${ }^{3)}$ Battistella et al. ${ }^{4)}$ showed that the attack lasted for less than 1 hour in 52\% of preschooler subjects, 1-2 hours in 20\%, 2-5 hours in $18 \%$, and $>5$ hours in $10 \%$. Raieli et al. ${ }^{7}$ suggested that $76.6 \%$ of the headache patients $<6$ years of age reported an attack duration of $<1$ hour, whereas $18.9 \%$ and $43.2 \%$ complained of pain for 1 hour and $<2$ hours, respectively. These studies support the results of this study on attack duration since almost half of the preschool patients reported having the attack for $<2$ hours and fewer subjects reported a duration of $>2$ hours.

Considering pain location in the current study, lateral areas were most commonly affected, followed by parietal and occipital areas. Virtanen et al. ${ }^{8}$ suggested that 6-year-old patients reported bilateral $(80 \%)$, forehead $(77 \%)$, above the eyes $(71 \%)$, unilateral $(24 \%)$, whole head (16\%), occipital (8\%), and temples (5\%) as the pain locations. Despite differences in options, the results were similar within the current study in the way that unilateral and bilateral pain was commonly reported compared to relatively less common pain in the occipital areas. The majority of patients in the current study reported having no accompanying symptoms. Nausea or vomiting was mentioned by patients with any related symptoms, followed by photophobia or phonophobia and dizziness. Battistella et al. ${ }^{4)}$ showed that 54\% of preschool patients reported phonophobia, followed by $52 \%$ with photophobia, $20 \%$ with nausea, and 18\% with vomiting. Similarly, Eidlitz-Markus et al. ${ }^{12)}$ suggested that photophobia (56\%) and phonophobia (54.3\%) were the most common associated symptoms in patients younger than 7 . They also reported that vomiting (44\%) and nausea (12.4\%) took the second rank, whereas only $1.9 \%$ of the patients experienced dizziness. The current study had some gaps compared to prior studies in terms of having a relatively smaller percentage of patients with these 2 associated symptoms, but the most patients had nausea or vomiting.

Topics such as pain location and related symptoms are quite subjective due to patients' level of ability to express their pain, but $>40 \%$ of patients could not explain their pain. Especially for related symptoms, preschoolers were unable to express themselves fluently, which makes their symptoms underestimated or differently estimated. They also might not be able to clearly understand some of the difficult symptoms of the questionnaires. Also, the answering rate of "unexplained" decreased considerably in attack frequency $(21.9 \%)$ or attack duration (30.9\%). This might have happened because those topics could be answered by parents who closely observed their young children. However, even these meticulous parents could not completely recognize attack frequency and duration since some significant moments were missing or forgotten. Finally, only 2 patients (1.4\%) could not explain the disease duration. This may imply that the disease duration is a relatively easier topic to answer broadly for both children and parents compared to other topics.

The average VAS score for pain severity was 5.1 \pm 2.2 , in which a score of 0 means no pain and a score of 10 stands for the most severe pain. Patients express pain severity using a specific number based on their thoughts or feelings. Despite VAS subjectivity, the answering rate (62.3\%) was relatively high among the topics for which only the patients themselves can provide an exact answer. Various facial expressions are accompanied by scores in the survey, which might help preschoolers understand the scale. ${ }^{13)}$ Mirshra et al. ${ }^{14}$ investigated headache severity using the VAS for children $>6$ years of age, and the Faces scale by the International Association for the Study of Pain was used for 3- to 6-year-old children. They suggested that 7.1\% of patients reported a pain degree as a score of $<3,31.0 \%$ reported a score of 3-5, 47.6\% had a score of 6-8, and 14.3\% had a score of 9-10. Although the exact data were not given, the average VAS score was derived as approximately $6.0 \pm 3.1$ using the median value of 
each score. ${ }^{14)}$ Small gaps in the score compared to the present study could be considered quite marginal if considering a wider range of subject age and smaller number of subjects.

A family history of headache was reported by $28.1 \%$ of patients in this study. Some articles suggested that a family headache history, especially maternal, is significantly correlated with headache in childhood. ${ }^{15)}$ Cuvellier et al. ${ }^{16)}$ showed that $73.5 \%$ of pediatric patients had a family history of headache, and the maternal history was the highest at $61.7 \%$. Cavestro et al. ${ }^{17)}$ suggested that $>90 \%$ of children aged 3-5 years with headache had a positive family history of headache similar to the findings of the study by Kroner-Herwig et al. ${ }^{18)}$ Also, environmental or social factors shared with family members could affect the occurrence or aggravation of childhood headache. Children with headaches seem to have fewer caring people $^{19)}$ and parents with a lower education level than children without headache. ${ }^{20)}$

None of the blood test findings was abnormal except in 1 patient with a clinically high level of TSH (12.62 $\mu \mathrm{IU} / \mathrm{mL})$ diagnosed with subclinical hypothyroidism. Hagen et al. ${ }^{21)}$ reported that headache was less probable in women with a TSH level $\geq 10 \mathrm{mU} / \mathrm{L}$ than in those with a normal TSH $(0.2 \pm 4 \mathrm{mU} / \mathrm{L})$. In contrast, Harbeck et al. $^{22)}$ suggested that a TSH deficiency was associated with fewer headaches than an adequate TSH level. Although controversial suggestions persist and only 1 patient in this study had an abnormal TSH level, there might be a correlation between headache and TSH level. Therefore, it would be meaningful if future studies could investigate the connections between them and include sufficient subjects with a high TSH level.

Brain MRI and EEG were used to identify the causes of headache, especially for secondary headaches such as congenital malformation. Park et al. ${ }^{23)}$ reported that 3 of 53 children had unusual results including subdural hematoma, cavernous hemangioma, or subarachnoid cyst. They also suggested that $17 \%$ of the patients had abnormal EEG outcomes including abnormal background activity (7.5\%), focal spike/sharp (7.5\%), or paroxysmal slow wave (1.9\%). In this study, sinusitis was considered as the cause of secondary headache, and with congenital abnormality, thought to be the cause of headache in about 12\% of all patients. Although only a few patients had positive findings in both studies, a future study including a number of patients with positive results on brain MRI and EEG would be helpful to aid our understanding of the characteristics of preschoolers with headache.

The most common headache-related diagnosis of children is migraine, followed by tension-type headache, similar to the diagnosis patterns seen in adults. ${ }^{3)}$ Mavromichalis et al. ${ }^{10)}$ suggested that $6.2 \%$ of 4- to 6-year-old patients had migraine, and the prevalence of migraine increased gradually with age as in other studies. ${ }^{3,10)}$ The results of this study similarly showed a tendency for the percentage of migraine or probable migraine to be the highest, followed by tension-type headache. In the current study, $71.2 \%$ of patients did not have specific diagnoses. Inclusion of data from a limited place and time might have caused this result; in addition, the ICHD 3-beta version has less diagnostic power for children.

Acetaminophen, ibuprofen, and naproxen sodium were used in a total of 26 patients in this study and are usually applied for children patients with acute headache. ${ }^{24)}$ Topiramate and amitriptyline were chosen as preventive medicines for 3 patients. According to Ahn and Shin, ${ }^{24)}$ these treatments are recommended if headache occurs more than once per a week or severe headache causing the child to be absent from school occurs more than once per month. Piazza et al. ${ }^{25)}$ reported that half of patients were treated with acute medication such as ketoprofen or paracetamol and $13.6 \%$ took a prophylactic pharmacological treatment. They suggested that medication overuse in headache therapy is a problem and that rational treatments are necessary to improve most patients. Regarding prognosis, more than half of patients improved, 3 underwent transfers to the department of neurosurgery, and 1 was transferred to the department of neurology. Tracing them, 2 patients showed repeated improvement and degeneration that resulted in subsequent hospital visits, while the other 2 were lost to follow-up. Kienbacher et al. ${ }^{26)}$ suggested that some factors such as female sex or longer follow-up period would positively affect the prognosis despite controversy and that early intervention might improve the prognosis of migraine and tensiontype headache in children and adolescents.

The present study has 2 limitations. First, prospective data accumulation would be required to decrease losses and ensure study accuracy. Also, we had difficulty finding the exact data needed in the retrospective medical chart reviews. The other limitation is that the current study was based on the data of patients at a single medical center during a relatively short period of time. Extending the record review period and performing consistent follow-up for each patient would enable the collection of broader data. It would also be of great help to collect additional patients by cooperating with other hospitals and then draw more universal conclusions.

The present study aimed to aid in the understanding and diagnosis of headache in childhood by investigating the various characteristics of preschoolers with headache. In summary, headache in preschool children has a relatively benign course, and diagnosis is possible according to ICHD 3-beta if history taking is detailed. In some cases, the cause of secondary headache can be identified through various investigations. In the future, more studies focusing on headache in this young age group are needed that will consider the features of children with headaches by age.

\section{Conflicts of interest}

No potential conflict of interest relevant to this article was reported. 


\section{Acknowledgments}

This work was supported by the research fund of Chungnam National University (2016-1516-01). This work was presented at the Korean Pediatric Society Congress in 2016.

\section{Supplementary material}

Supplementary Table can be found via https://doi.org/10.3345/ kjp.2018.06331.

\section{References}

1. Albuquerque RP, Santos AB, Tognola WA, Arruda MA. An epidemiologic study of headaches in Brazilian schoolchildren with a focus on pain frequency. Arq Neuropsiquiatr 2009;67(3B):798-803.

2. Lanzi G, D'Arrigo S, Termine C, Rossi M, Ferrari-Ginevra O, Mongelli A, et al. The effectiveness of hospitalization in the treatment of paediatric idiopathic headache patients. Psychopathology 2007;40:1-7.

3. Hong YJ, Kim MS, Lee KY, Sim CS. Clinical characteristics of primary headache according to age in children and adolescents. J Korean Child Neurol Soc 2010;18:264-74.

4. Battistella PA, Fiumana E, Binelli M, Bertossi E, Battista P, Perakis E, et al. Primary headaches in preschool age children: clinical study and follow-up in 163 patients. Cephalalgia 2006;26:162-71.

5. Sillanpaa M. Prevalence of migraine and other headache in Finnish children starting school. Headache 1976;15:288-90.

6. Lee KK. Differential diagnosis and management of headaches. Korean J Fam Practice 2013;3:255-9.

7. Raieli V, Eliseo M, Pandolfi E, La Vecchia M, La Franca G, Puma D, et al. Recurrent and chronic headaches in children below six years of age. J Headache Pain 2005;6:135-42.

8. Virtanen R, Aromaa M, Rautava P, Metsahonkala L, Anttila P, Helenius $\mathrm{H}$, et al. Changing headache from preschool age to puberty. A controlled study. Cephalalgia 2007;27:294-303.

9. Hernandez-Latorre MA, Roig M. Natural history of migraine in childhood. Cephalalgia 2000;20:573-9.

10. Mavromichalis I, Anagnostopoulos D, Metaxas N, Papanastassiou E. Prevalence of migraine in schoolchildren and some clinical comparisons between migraine with and without aura. Headache 1999;39: 728-36.

11. Laurell K, Larsson B, Eeg-Olofsson O. Prevalence of headache in Swedish schoolchildren, with a focus on tension-type headache. Cephalal- gia 2004;24:380-8.

12. Eidlitz-Markus T, Gorali O, Haimi-Cohen Y, Zeharia A. Symptoms of migraine in the paediatric population by age group. Cephalalgia 2008; 28:1259-63.

13. Wewers ME, Lowe NK. A critical review of visual analogue scales in the measurement of clinical phenomena. Res Nurs Health 1990;13: 227-36.

14. Mishra D, Sharma A, Juneja M, Singh K. Recurrent headache in pediatric outpatients at public hospital in Delhi. Indian Pediatr 2013; 50:775-8.

15. Na HJ, Kim JH, Park SY, Han JY, Eom TH, Bin JH, et al. Effects of gender, age, family history and shoulder pain on pediatric primary headache. J Korean Child Neurol Soc 2015;23:27-30.

16. Cuvellier JC, Donnet A, Guegan-Massardier E, Nachit-Ouinekh F, Parain D, Vallee L, et al. Clinical features of primary headache in children: a multicentre hospital-based study in France. Cephalalgia 2008;28:1145-53.

17. Cavestro C, Montrucchio F, Benci P, Pompilio D, Mandrino S, Cencio PG, et al. Headache prevalence and related symptoms, family history, and treatment habits in a representative population of children in Alba, Italy. Ped Neurol 2014;51:348-53.

18. Kroner-Herwig B, Heinrich M, Morris L. Headache in German children and adolescents: a population-based epidemiological study. Cephalalgia 2007;27:519-27.

19. Carisson J. Prevalence of headache in schoolchildren: relation to family and school factors. Acta Paediatrica 1998;85:692-6.

20. Metsähonkala L, Sillanpää M, Tuominen J. Social environment and headache in 8- to 9-Year-Old Childen: a follow-up study. Headache 1998;38:222-8.

21. Hagen K, Bjùro T, Zwart JA, Vatten L, Stovner LJ, Bovim G. Low headache prevalence amongst women with high TSH values. Eur J Neurol 2001;8:693-9.

22. Harbeck B, Haas CS, Suefke S, Kropp P, Moenig H. Headache and depression in patients with hypothalamic-pituitary disorders-etiology and risk factors. Exper Clin Endocrinol Diabetes 2015;123:571-4.

23. Park SS, Bae GY, Kim EJ, Hwang KK. A clinical study of recurrent headaches in children and an application of International Headache Society Classification to children. J Korean Child Neurol Soc 1997; 5:95-105.

24. Ahn HS, Shin HY. Hong Chang-Eui pediatrics. Seoul: Mirae N, 2016: 1104-8.

25. Piazza F, Chiappedi M, Maffioletti E, Galli F, Balottin U. Medication overuse headache in school-aged children: more common than expected? Headache 2012;52:1506-10.

26. Kienbacher C, Wober C, Zesch HE, Hafferl-Gattermayer A, Posch M, Karwautz A, et al. Clinical features, classification and prognosis of migraine and tension-type headache in children and adolescents: a long-term follow-up study. Cephalalgia 2006;26:820-30. 